\title{
High efficient luminescence in type-II GaAsSb-capped InAs quantum dots upon annealing
}

\author{
J. M. Ulloa, ${ }^{1, a)}$ J. M. Llorens, ${ }^{2}$ B. Alén, ${ }^{2}$ D. F. Reyes, ${ }^{3}$ D. L. Sales, ${ }^{3}$ D. González, ${ }^{3}$ \\ and A. Hierro ${ }^{1}$ \\ ${ }^{1}$ Institute for Systems based on Optoelectronics and Microtechnology (ISOM), Universidad Politecnica de \\ Madrid, Ciudad Universitaria s/n, 28040 Madrid, Spain \\ ${ }^{2}$ IMM-Instituto de Microelectrónica de Madrid (CNM-CSIC), Isaac Newton 8, PTM, E-28760 Tres Cantos, \\ Madrid, Spain \\ ${ }^{3}$ Departamento de Ciencia de los Materiales e IM y QI, Universidad de Cádiz, 11510 Puerto Real (Cádiz), \\ Spain
}

(Received 6 November 2012; accepted 7 December 2012; published online 21 December 2012)

\begin{abstract}
The photoluminescence efficiency of GaAsSb-capped InAs/GaAs type II quantum dots (QDs) can be greatly enhanced by rapid thermal annealing while preserving long radiative lifetimes which are 20 times larger than in standard GaAs-capped InAs/GaAs QDs. Despite the reduced electron-hole wavefunction overlap, the type-II samples are more efficient than the type-I counterparts in terms of luminescence, showing a great potential for device applications. Strain-driven In-Ga intermixing during annealing is found to modify the QD shape and composition, while As-Sb exchange is inhibited, allowing to keep the type-II structure. $\mathrm{Sb}$ is only redistributed within the capping layer giving rise to a more homogeneous composition. (C) 2012 American Institute of Physics.

[http://dx.doi.org/10.1063/1.4773008]
\end{abstract}

Semiconductor quantum dots (QDs) with a type-II band alignment, in which either electrons or holes are located outside the QD, have recently attracted a lot of interest due to possible advantages over the commonly used type-I QDs in some applications. Indeed, the spatial separation of electrons and holes makes them good candidates for the realization of QD memories ${ }^{1,2}$ or QD solar cells. ${ }^{3}$ Light sources with typeII QDs might also have advantages, such as wavelength and gain tunability. ${ }^{4-6}$ Moreover, type-II QD lasers could present an inhibition of spontaneous emission and Auger recombination, which may result in a reduced threshold current, as it has been predicted for type-II quantum well (QW) lasers. ${ }^{6,7}$ However, the spatial separation of electrons and holes is also responsible for their weak emission efficiency, which is one of the major drawbacks for the application of type II QDs. The photoluminescence (PL) efficiency of GaSb/GaAs QDs, probably the most studied III-V type-II QD system, is typically much weaker than that of standard type-I QDs grown on GaAs, and very often the emission from the QDs is even weaker than the emission of the wetting layer (WL). ${ }^{8,9}$ For this reason, there have been almost no reports on type-II QD lasers, ${ }^{4,10}$ although many groups have demonstrated lasing from type-II QWs. ${ }^{11,12}$ Therefore, III-V type-II QDs with good optical properties are still required to fully exploit their advantages in device applications.

In this paper, we demonstrate a GaAs-based type-II QD system with a PL emission that is narrower and more intense than that of standard type-I InAs/GaAs QDs. We show that the emission efficiency of GaAsSb-capped InAs/GaAs type II QDs can be greatly enhanced by rapid thermal annealing (RTA) while preserving the long radiative lifetimes. The structural changes induced by the RTA process are eluci-

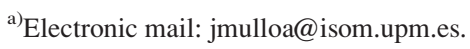

dated by a combination of transmission electron microscopy (TEM) and $8 \times 8 \mathrm{k} \cdot \mathrm{p}$ modeling.

The two analyzed samples were grown by solid source molecular beam epitaxy (MBE) on Si doped (100) $n^{+}$GaAs substrates. The QDs were grown under the same conditions in both samples by depositing 2.7 monolayers (MLs) of InAs at $450{ }^{\circ} \mathrm{C}$ and $0.04 \mathrm{ML} / \mathrm{s}$ on an intrinsic GaAs buffer layer. After $20 \mathrm{~s}$ of growth interruption, the QD layer was capped with $20 \mathrm{MLs}$ of GaAs or GaAsSb grown at $470{ }^{\circ} \mathrm{C}$ (hereafter, called samples Ref and $\mathrm{Sb}$, respectively). Both samples were subsequently covered with $250 \mathrm{~nm}$ of GaAs grown at $580^{\circ} \mathrm{C}$. The nominal Sb content in the GaAsSb-capped QD sample was $28 \%$.

Time integrated PL was measured at $15 \mathrm{~K}$ using a closed cycle helium cryostat and a He-Ne laser as the excitation source. The emitted light was dispersed through a $1 \mathrm{~m}$ spectrometer and detected with a liquid nitrogen-cooled Ge detector using standard lock-in techniques. Time resolved PL (TRPL) transients were measured at $20 \mathrm{~K}$ using time correlated single photon counting electronics. TRPL was excited with a $405 \mathrm{~nm}$ pulsed diode laser (pulse duration $70 \mathrm{ps}$ ) and detected with a fast InGaAs photomultiplier (transit time spread $400 \mathrm{ps}$ ) attached to a $0.3 \mathrm{~m}$ monochromator. The average excitation power density was kept low $\left(0.6 \mathrm{~W} / \mathrm{cm}^{2}\right.$ at $10 \mathrm{MHz}$ ) to minimize charge accumulation effects that might lead to emission blue shifts in type II heterostructures. TEM measurements were performed on a JEOL-2011 and a JEOL$2010 \mathrm{~F}$, equipped with a field-emission gun and operating at $200 \mathrm{kV}$. RTA under nitrogen atmosphere was performed using a MILA-3000 oven. The samples were capped with a GaAs wafer during the process.

Introducing a thin GaAsSb capping layer not only induces a strong red shift of the PL peak of InAs/GaAs QDs but also increases the inhomogeneous broadening and reduces the integrated intensity, as can be inferred from the 
continuous line spectra in Fig. 1(a). The high Sb content guarantees a type-II band alignment, with the holes localized outside the QD in the capping layer. ${ }^{13,14}$ This is evident from the time resolved PL measurements, in which a much longer carrier lifetime is obtained for the Sb-containing sample. Fig. 1(b) displays the TRPL decay transients measured at the maximum of the spectra for the two as-grown samples. The measurements can be fitted to a double exponential decay function after convolution with the instrument response function (IRF) shown in the same graph. Two decay lifetimes with different relative weights (decay time-amplitude products) are, therefore, obtained for each sample and shown in the inset table in Fig. 1(b). In sample Ref (GaAs-capped QDs), a typical radiative recombination lifetime of $0.7 \mathrm{~ns}$ is retrieved, with a small contribution of a slower component ( $5.4 \mathrm{~ns}$ and weight of $4.5 \%$ ) which can be attributed to dark exciton recombination. The situation is quite different in sample $\mathrm{Sb}$ (GaAsSb-capped QDs), in which a much larger lifetime of $14.2 \mathrm{~ns}$ dominates carrier recombination (with a weight of $86.9 \%$ ). This is indicative of a type-II band alignment, in which the reduced electron-hole wavefunction overlap gives rise to a large radiative lifetime. ${ }^{15}$ The small and
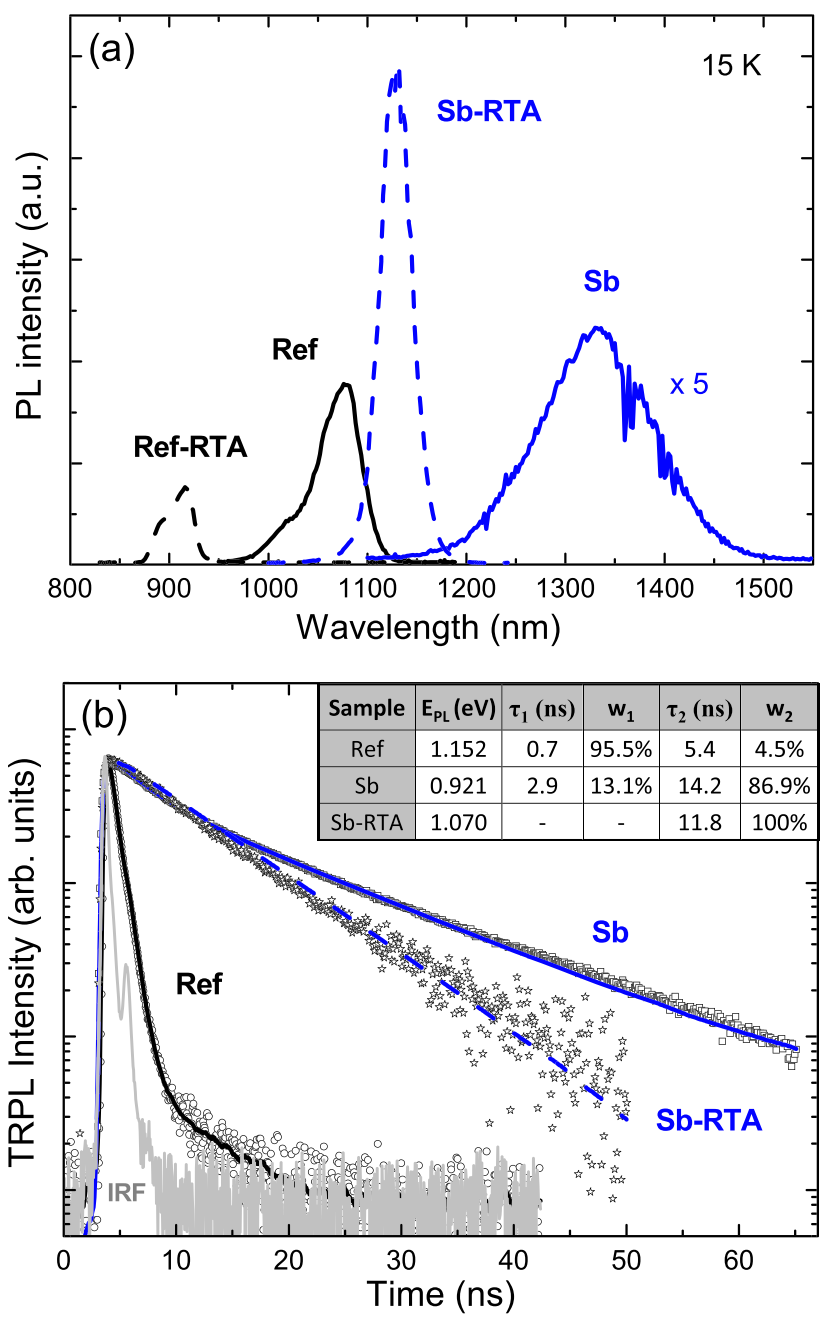

FIG. 1. (a) $15 \mathrm{~K}$ PL spectra of samples Ref, Ref-RTA, Sb, and Sb-RTA. (b) PL decay transients of samples Ref, Sb, and Sb-RTA measured at the PL maximum. The IRF was measured with an ultrafast laser diode emitting at $950 \mathrm{~nm}$. The inset table shows the PL peak energy and the obtained radiative lifetimes with the corresponding weights for the three samples. faster component should be associated in this case to defective QDs which suffer of non-radiative recombination in parallel with the radiative channels.

A RTA cycle at $850^{\circ} \mathrm{C}$ during $30 \mathrm{~s}$ was performed to both samples (from now on called samples Ref-RTA and Sb-RTA). The PL of the annealed samples is also shown in Fig. 1(a) (dashed line spectra). A very strong improvement of the PL emission was obtained for the Sb-RTA sample, with an increase of the integrated intensity by a factor of 3.3 (the peak intensity increases by a factor of 10) and a reduction of the full width at half maximum (FWHM) of $67 \%$, from $97 \mathrm{meV}$ down to $32 \mathrm{meV}$. A blue shift of the peak wavelength of $149 \mathrm{meV}$ is also observed. The situation is quite different than in sample Ref-RTA, in which a reduction of the integrated intensity and a $2 \mathrm{meV}$ increase of the FWHM is observed.

One could be tempted, at first sight, to attribute the improvement in sample $\mathrm{Sb}$ to the transition from a type-II to a type-I band alignment due to intermixing processes taking place during annealing. Indeed, this has recently been reported for similar structures. ${ }^{16}$ Nevertheless, time resolved PL shows that this is not the case (Fig. 1(b)) since a single exponential decay at the peak of the spectra of sample SbRTA is found with a long lifetime of $11.8 \mathrm{~ns}$. An analysis of the decay time with temperature (not shown) reveals that thermally activated non-radiative channels are negligible below $140 \mathrm{~K}$. Therefore, at low temperatures, the radiative lifetime after annealing is still very similar to that in the asgrown sample and 17 times higher than that in the type-I sample Ref. This means that the band alignment remains type-II, contrary to what has been previously reported. ${ }^{16}$ As abovementioned, the PL of the annealed sample is much more efficient than that of the as-grown. Indeed, the PL of the Sb-RTA sample is more intense than that of the standard GaAs-capped QDs before and after annealing, which is a striking result taking into account the type-I alignment of the latter and the similar QD density found in both samples. This demonstrates that a type-II QD system with long radiative lifetime can compete with type-I QD systems in terms of PL efficiency, and therefore its particularities can be exploited for applications in devices.

In order to clarify the structural changes that take place during the annealing process, TEM measurements were performed on samples $\mathrm{Sb}$ and Sb-RTA. TEM images acquired on g002 dark field mode of both samples are shown in Figs. 2(a) and 2(b). Several QDs, the WL with darker contrast with respect to GaAs, and the capping layer (brighter contrast), are clearly distinguished in both images. The average size and shape of the QDs were determined after analyzing up to 60 QDs in both samples. In addition, maps of the strain along the growth direction $\left(\varepsilon_{z z}\right)$ were determined from high resolution TEM images acquired on the [110] pole axis of regions away from QDs (not shown). From the analysis of the strain data, the average $\mathrm{Sb}$ content in both samples was determined using the same procedure described in Ref. 17. The main conclusions of the TEM analysis are the following:

(1) The QDs become flatter (more quantum disk-like) after annealing. The average QD height is reduced by $1 \mathrm{~nm}$ (from 3.3 to $2.3 \mathrm{~nm}$, see Fig. 2(c)) and the average base diameter increases by $6 \mathrm{~nm}$ (from 16 to $22 \mathrm{~nm}$ ). 

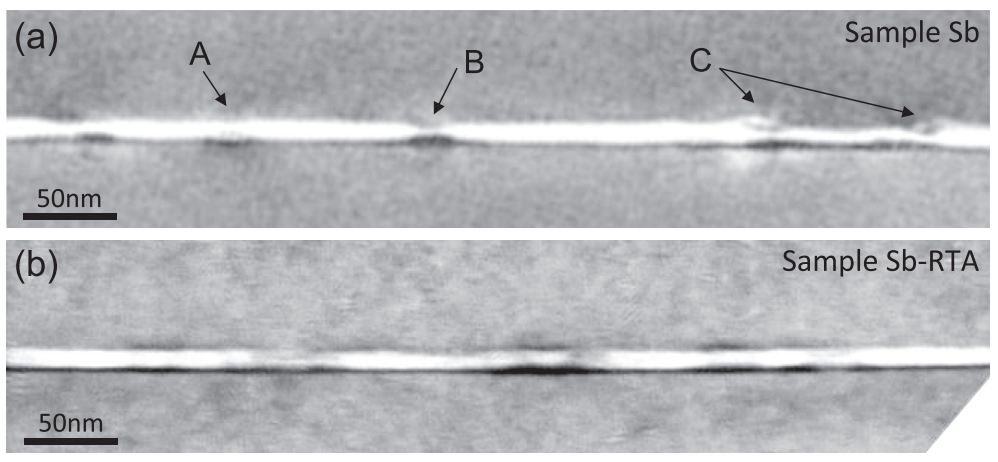

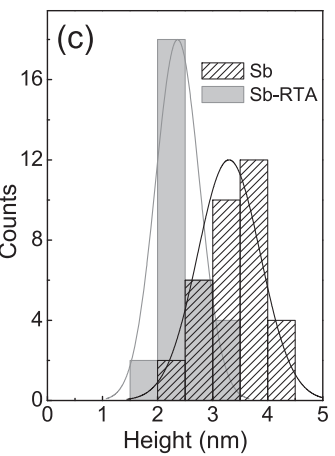

FIG. 2. g002 dark field TEM images of samples Sb (a) and Sb-RTA (b). (c) Shows the measured QD height distribution in both samples.

(2) The QD height distribution becomes narrower after annealing, as shown in Fig. 2(c) (the standard deviation decreases from 0.57 to $0.39 \mathrm{~nm}$ ).

(3) The thickness of the capping layer decreases $\sim 1 \mathrm{~nm}$ after annealing, from 6.5 to $5.5 \mathrm{~nm}$, which is likely due to $\mathrm{Sb}$ segregation of the top monolayers of the GaAsSb capping layer in the growth direction.

(4) The Sb content in the capping layer far from the QDs remains unchanged and equal to $20 \pm 1 \%$ (slightly lower than the nominal value).

(5) There is a significant inhomogeneity in the regions of the capping layer over the QDs in sample Sb. While the capping layer perfectly follows the shape of the QDs in some cases (see feature A in Fig. 2(a)), part of the capping layer is missing on top of some QDs, giving rise to a deep basin (see feature B in Fig. 2(a)) or to an altered capping structure (feature C in Fig. 2(a)). Similar features have been previously observed in related samples, and the morphology of the GaAsSb capping layer on top of the QDs was shown to be very sensitive to the growth temperature. ${ }^{18}$ This effect disappears after annealing, since no deep features are observed on top of the QDs in the Sb-RTA sample.

The reduced QD height distribution and inhomogeneity of the capping layer on top of the QDs are consistent with the reduced inhomogeneous broadening and FWHM observed after annealing. However, to explain most of the emission properties of the annealed Sb-RTA sample, and, in particular, its large blue shift and its type II confinement character, a theoretical calculation of the QD electronic states must be combined with the TEM measurements. The NEXTNANO ++ software package $^{19}$ was used for calculating the electronic structure in samples $\mathrm{Sb}$ and Sb-RTA. The theoretical model is based on the $8 \times 8 \mathrm{k} \cdot \mathrm{p}$ method, including the inhomogeneous strain distribution and its associated linear piezoelectric field. The Schrödinger equation was solved in a simulation box extending $10 \mathrm{~nm}$ farther apart from the QD. The continuum elastic and Poisson equations were solved inside a larger box extending $50 \mathrm{~nm}$ away from the QD. In all cases, Dirichlet boundary conditions were assumed. The empirical band parameters were taken from Ref. 20. A realistic structure was simulated, in which the input QD size and shape, as well as the capping layer thickness and $\mathrm{Sb}$ content were obtained from the TEM measurements, and only the Ga content inside the QDs was considered as parameter to be explored. We find that introducing the reduced QD height, we can partly explain the observed blue shift, but some In-Ga intermixing processes between the QD and capping layer must be also introduced to fully explain our results. In-Ga intermixing is well known for GaAs-capped QDs which become more Ga-rich upon annealing leading to the strong blue shifts observed here for the Ref sample. ${ }^{21}$ The effect of such In-Ga intermixing processes in GaAsSb capped QDs is less understood as the final result might depend also on the role played by $\mathrm{Sb}-\mathrm{As}$ exchange between the capping layer and the QD.

Figure 3(a) shows the calculated fundamental transition energy for the as-grown sample $\mathrm{Sb}$ (full red dot). The best fit to the measured PL peak energy is obtained when a pure InAs QD is considered. The calculated band structure is shown in Fig. 3(b). The continuous black lines show the band edge potential profiles across the center of the QD. Nevertheless, the potential minimum for holes is not located in the center of the QD. The ground state hole probability density in the (1-10) plane (left hand side inset in Fig. 3(a)) shows that the potential minimum is split in two parts located outside the QD close to the QD-capping layer interface in the [110] direction, as predicted recently for similar structures. ${ }^{22,23}$ The dashed blue line shows the profile along the potential minimum, where the maximum of the probability density of the hole wavefunction is located. If the transition energy for the annealed sample is calculated considering the same QD composition than in the as-grown $(0 \% \mathrm{Ga})$, a small blue shift of $19 \mathrm{meV}$ is obtained for the fundamental transition energy (see Fig. 3(a)). This is the blue shift induced by the reduced QD height, which is clearly much smaller than the measured one. Since our TEM analysis reveals that the $\mathrm{Sb}$ content in the capping layer remains unmodified after annealing, the extra blue shift must likely be due to an increase of the Ga content inside the QD as a result of In-Ga intermixing. Fig. 3(a) shows how the fundamental transition energy increases when the Ga content in the QD is increased. Increasing Ga has a strong impact on the confined energy levels due to both the increased band gap of the alloy and the reduced strain in the QD. To match the experimental blue shift, a Ga content of $27 \%$ inside the QD has to be considered. This shows that strong In-Ga intermixing takes place during the RTA process, modifying considerably the QD composition. Most remarkably, the proposed mechanism keeps the type-II alignment unaltered (see Fig. 3(c) and the right-hand side inset in Fig. 3(a)), 

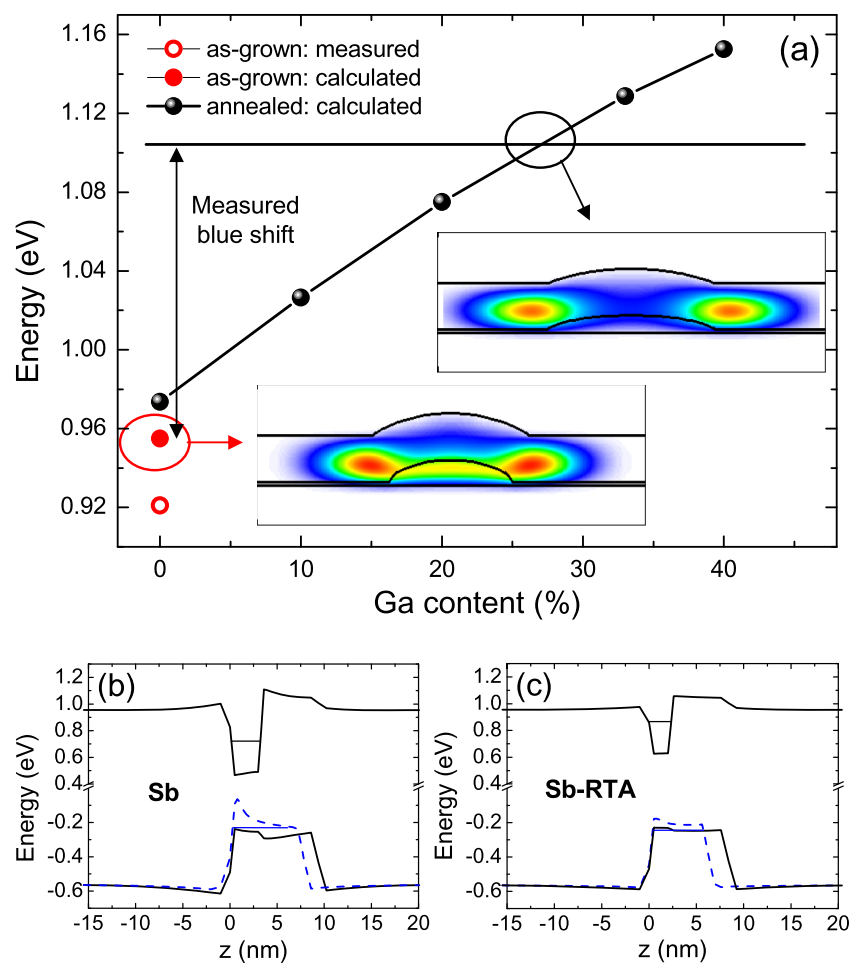

FIG. 3. (a) Energy of the PL maximum and of the calculated fundamental transition of sample Sb considering 100\% In inside the QDs (empty and solid red spot, respectively). Calculated energy of the fundamental transition for sample Sb-RTA as a function of the Ga content in the QDs (in black). In order to reproduce the measured PL blue shift, a Ga concentration of $27 \%$ inside the QD must be considered. The insets show the ground state hole probability density in the (1-10) plane for samples Sb and Sb-RTA. (b) and (c) show the calculated band edge layout for samples Sb and Sb-RTA, respectively. The continuous black lines are the band edge profiles across the center of the QD. The dashed blue lines are the band edge profiles across the potential minimum, where the maximum of the probability density of the hole wavefunction is located.

leaving the hole ground state located outside the QD close to the QD-capping layer interface in the [110] direction. This nicely agrees with the results from the time resolved PL measurements.

The calculated radiative lifetimes (goes as the inverse of the oscillator strength $)^{23}$ are $11 \mathrm{~ns}$ and $6 \mathrm{~ns}$ for samples $\mathrm{Sb}$ and Sb-RTA, respectively. These values fit reasonably well with the experimental results. The calculated decrease of $\tau$ after annealing is the result of the stronger electron-hole wavefunction overlap in sample Sb-RTA. This is due to the increased electron wavefunction delocalization to the capping layer resulting from the increased energy of the electron ground state induced by the reduced QD height and the intermixing. Although the measured decrease of the radiative lifetime after annealing is smaller than the one predicted, this could contribute to the observed enhancement of the PL integrated intensity after annealing.

A complete picture of the ongoing processes during annealing can now be drawn. On one hand, a strong In-Ga intermixing between the QD and capping layer takes place during annealing, while As-Sb exchange is inhibited ( $\mathrm{Sb}$ only segregates slightly in the growth direction and rearranges itself within the capping layer giving rise to a more homogeneous Sb distribution above the QDs). It is the absence of As-Sb exchange between the QD and capping layer which allows maintaining the type-II band alignment. The driving force behind this is likely strain minimization ${ }^{21}$ (Ga incorporation into the QD reduces the strain, while $\mathrm{Sb}$ incorporation would increase the QD strain). In-Ga intermixing results in a flattening and $\mathrm{a} \sim 27 \%$ Ga enrichment of the QDs, which explains the strong blue shift observed on the PL. According to the model, the average in-plain strain components $\varepsilon_{\mathrm{xx}}=\varepsilon_{\mathrm{yy}}$ in the QD decrease from $-6.0 \%$ to $-4.7 \%$ in the new structure, and the $\varepsilon_{\mathrm{zz}}$ component from $4.1 \%$ to $4.0 \%$. The actual reduction of the average elastic energy in the QD is $16 \%$, which supports the idea of strain minimization as the driving force of the ongoing processes during RTA. The modified shape and composition contribute to increase the electron-hole wavefunction overlap (it must be noticed that this is the case only because of the type-II alignment). Moreover, QDs that were initially optically inactive or subject to non-radiative channels are likely becoming active upon annealing, which could contribute to the increased PL integrated intensity. The fact that the fast component of the carrier lifetime, likely related to nonradiative channels, disappears after annealing (see the inset in Fig. 1(b)) supports this idea. In-Ga intermixing results also in a QD size and shape homogenization, which together with the Sb distribution homogenization in the capping layer on top of the QDs would explain the strong reduction in the PL inhomogeneous broadening. All this together allows type-II GaAsSb-capped InAs QDs to become even more efficient in terms of PL than standard type-I InAs QDs, which makes them a main candidate to exploit the possibilities of type-II QDs in device applications.

This work has been supported by Spanish MICINN (Project No. MAT2010-15206), MEC (Project No. TEC2011-29120-C05-04), and CAM (Project No. P2009/ ESP-1503). J. M. Ulloa was supported by MICINN through the "Ramón y Cajal" program. TEM measurements were taken at DME-SCCYT-UCA.

${ }^{1}$ M. Geller, C. Kapteyn, L. Müller-Kirsch, R. Heitz, and D. Bimberg, Phys. Status Solidi B 238, 258 (2003).

${ }^{2}$ A. Marent, M. Geller, A. Schliwa, D. Feise, K. Pötschke, D. Bimberg, N. Akçay, and N. Öncan, Appl. Phys. Lett. 91, 242109 (2007).

${ }^{3}$ R. B. Laghumavarapu, A. Moscho, A. Khoshakhlagh, M. El-Emawy, L. F. Lester, and D. L. Huffaker, Appl. Phys. Lett. 90, 173125 (2007).

${ }^{4}$ J. Tatebayashi, A. Khoshakhlagh, S. H. Huang, G. Balakrishnan, L. R. Dawson, D. L. Huffaker, D. A. Bussian, H. Htoon, and V. Klimov, Appl. Phys. Lett. 90, 261115 (2007).

${ }^{5}$ M. Montes Bajo, J. M. Ulloa, M. del Moral, Á. Guzmán, and A. Hierro, IEEE J. Quantum Electron. 47, 1547 (2011).

${ }^{6}$ W. W. Chow and H. C. Schneider, Appl. Phys. Lett. 78, 4100 (2001).

${ }^{7}$ J. R. Meyer, C. A. Hoffman, F. J. Bartoli, and L. R. Ram-Mohan, Appl. Phys. Lett. 67, 757 (1995).

${ }^{8}$ K. Suzuki, R. A. Hogg, and I. Arakawa, J. Appl. Phys. 85, 8349 (1999).

${ }^{9}$ C. Y. Jin, H. Y. Liu, S. Y. Zhang, Q. Jiang, S. L. Liew, M. Hopkinson, T. J. Badcock, E. Nabavi, and D. J. Mowbray, Appl. Phys. Lett. 91, 021102 (2007).

${ }^{10}$ K. S. Hsu, T. T. Chiu, W.-H. Lin, K. L. Chen, M. H. Shih, S.-Y. Lin, and Y.-C. Chang, Appl. Phys. Lett. 98, 051105 (2011).

${ }^{11}$ C. L. Felix, J. R. Meyer, I. Vurgaftman, C.-H. Lin, S. J. Murry, D. Zhang, and S.-S. Pei, IEEE Photonics Technol. Lett. 9, 734 (1997).

${ }^{12}$ R. Kaspi, A. Ongstad, G. C. Dente, J. Chavez, M. L. Tilton, and D. Gianardi, Appl. Phys. Lett. 81, 406 (2002).

${ }^{13}$ H. Y. Liu, M. J. Steer, T. J. Badcock, D. J. Mowbray, M. S. Skolnick, F. Suarez, J. S. Ng, M. Hopkinson, and J. P. R. David, J. Appl. Phys. 99, 046104 (2006).

${ }^{14}$ J. M. Ulloa, R. Gargallo-Caballero, M. Bozkurt, M. del Moral, A. Guzmán, P. M. Koenraad, and A. Hierro, Phys. Rev. B 81, 165305 (2010). 
${ }^{15}$ Y. D. Jang, T. J. Badcock, D. J. Mowbray, M. S. Skolnick, J. Park, D. Lee, H. Y. Liu, M. J. Steer, and M. Hopkinson, Appl. Phys. Lett. 92, 251905 (2008).

${ }^{16}$ Y.-A. Liao, W.-T. Hsu, P.-C. Chiu, J.-I. Chyi, and W.-H. Chang, Appl. Phys. Lett. 94, 053101 (2009).

${ }^{17}$ J. M. Ulloa, D. F. Reyes, M. Montes, K. Yamamoto, D. L. Sales, D. Gonzalez, A. Guzman, and A. Hierro, Appl. Phys. Lett. 100, 013107 (2012).

${ }^{18}$ J. M. Ulloa, I. W. D. Drouzas, P. M. Koenraad, D. J. Mowbray, M. J. Steer, H. Y. Liu, and M. Hopkinson, Appl. Phys. Lett. 90, 213105 (2007).
${ }^{19}$ S. Birner, T. Zibold, T. Andlauer, T. Kubis, M. Sabathil, A. Trellakis, and P. Vogl, IEEE Trans. Electron Devices 54, 2137 (2007).

${ }^{20}$ A. Babiński, J. Jasiński, R. Bożek, A. Szepielow, and J. M. Baranowski, Appl. Phys. Lett. 79, 2576 (2001).

${ }^{21}$ I. Vurgaftman, J. R. Meyer, and L. R. Ram-Mohan, J. Appl. Phys. 89, 5815 (2001).

${ }^{22}$ P. Klenovsky, V. Krapek, D. Munzar, and J. Humlicek, Appl. Phys. Lett. 97, 203107 (2010).

${ }^{23}$ J. M. Ulloa, J. M. Llorens, M. del Moral, M. Bozkurt, P. M. Koenraad, and A. Hierro, J. Appl. Phys. 112, 074311 (2012). 